\title{
Turbulent Diffusion of Lines and Circulations
}

\author{
Gregory L. Eyink \\ Department of Applied Mathematics \& Statistics \\ The Johns Hopkins University \\ 3400 N. Charles Street \\ Baltimore, MD 21218 \\ Tel: 410-516-7201, Fax: 410-516-7459 \\ e-mail: eyink@ams.jhu.edu
}

\begin{abstract}
We study material lines and passive vectors in a model of turbulent flow at infiniteReynolds number, the Kraichnan-Kazantsev ensemble of velocities that are white-noise in time and rough (Hölder continuous) in space. It is argued that the phenomenon of "spontaneous stochasticity" generalizes to material lines and that conservation of circulations generalizes to a "martingale property" of the stochastic process of lines.

PACS: 47.27.Jv, 52.65.Kj, 02.50.Fz, 05.45.Df

keywords: turbulence, material lines, circulations, Kraichnan model, dynamo, fractals
\end{abstract}


The evolution of material lines and surfaces passively carried by turbulent flow has long been a subject of interest [1]. This is motivated in part by questions surrounding dynamically relevant objects, such as vortex lines [2, 3, 4] and magnetic field-lines [5, 6], which have been argued to behave similar to material lines at high Reynolds numbers. However, in that limit, the turbulent velocity field is no longer differentiable in space but only Hölder continuous [7, 8, 9]. Observations from experiments and simulations suggest that material objects advected by such a rough velocity become fractal, with a Hausdorff dimension strictly greater than their topological dimension [10, 11, 12, 13, 14, 15]. This poses a difficulty to the view that vortex lines behave as material lines - a consequence of the Kelvin-Helmholtz theorem [16, 17]-since circulations are a priori not defined for non-rectifiable loops. It has recently been argued that the Kelvin theorem in fact breaks down in turbulent flows, in the sense that the circulation is not strictly conserved for every loop [18, 19]. Similar breakdown of Alfvén's theorem on magnetic-flux conservation [20] is expected in plasma turbulence at high magnetic Reynolds numbers [21].

These questions have been sharpened by recent work on the Kraichnan model of advection by a Gaussian random velocity field that is delta-correlated in time [22]. A novel phenomenon has been discovered there called spontaneous stochasticity: Lagrangian particle trajectories for a non-Lipschitz advecting velocity are non-unique and split to form a random process in pathspace for a fixed velocity realization [23, 24, 25, 26, 27, 28, 29]. This phenomenon raises many fundamental questions, including whether material objects such as lines and surfaces can even exist in the limit of infinite Reynolds number. It is the purpose of this Letter to outline a new approach to the evolution of such geometric objects in the Kraichnan model. We focus on material lines and passive vectors, which are dual objects in the same sense as material particles and passive scalar fields [30]. In particular, we shall sketch the proof of a "martingale property" that has previously been proposed [18] as a generalization of the conservation of circulations for a rough velocity field.

We consider stochastic flows [31] on a $d$-dimensional manifold $\mathcal{M}$ driven by Brownian vector fields that are not Lipschitz regular in space. To simplify the presentation, we use Euclidean 
space $\mathcal{M}=\mathbb{R}^{d}$ or the torus $\mathcal{M}=\mathbb{T}^{d}$ to illustrate the main ideas. More precisely, $\mathbf{u}(\mathbf{x}, t)$ is a Gaussian random vector field, with mean $\overline{\mathbf{u}}(\mathbf{x}, t)$ and fluctuating part $\widetilde{\mathbf{u}}(\mathbf{x}, t)$ with covariance

$$
\left\langle\widetilde{u}_{i}(\mathbf{x}, t) \widetilde{u}_{j}\left(\mathbf{x}^{\prime}, t^{\prime}\right)\right\rangle=D_{i j}\left(\mathbf{x}, \mathbf{x}^{\prime} ; t\right) \delta\left(t-t^{\prime}\right)
$$

for $\mathbf{x}, \mathbf{x}^{\prime} \in \mathcal{M}$. We are mainly interested in the case that $\overline{\mathbf{u}}(\mathbf{x}, t) \equiv \mathbf{0}$ and $\mathbf{u}(\mathbf{x}, t) \equiv \widetilde{\mathbf{u}}(\mathbf{x}, t)$ is a homogeneous random field, with $\mathbf{D}\left(\mathbf{x}, \mathbf{x}^{\prime} ; t\right)=\mathbf{D}\left(\mathbf{x}-\mathbf{x}^{\prime}, t\right)$. The quantity

$$
\Delta\left(\mathbf{x}, \mathbf{x}^{\prime} ; t\right)=\operatorname{tr}\left[\mathbf{D}(\mathbf{x}, \mathbf{x} ; t)+\mathbf{D}\left(\mathbf{x}^{\prime}, \mathbf{x}^{\prime} ; t\right)-2 \mathbf{D}\left(\mathbf{x}, \mathbf{x}^{\prime} ; t\right)\right]
$$

is $\left\langle\left\|\mathbf{u}(\mathbf{x})-\mathbf{u}\left(\mathbf{x}^{\prime}\right)\right\|^{2}\right\rangle$, the mean of the Euclidean norm squared, for a random field $\mathbf{u}(\mathbf{x})$ with covariance $\mathbf{D}\left(\mathbf{x}, \mathbf{x}^{\prime} ; t\right)$. The case of greatest interest to us has $\Delta\left(\mathbf{x}, \mathbf{x}^{\prime} ; t\right) \propto\left\|\mathbf{x}-\mathbf{x}^{\prime}\right\|^{2 \alpha}$ with $0<\alpha<1$. In that case, $\mathbf{u}(\mathbf{x})$ is Hölder continuous with exponent $\alpha$ at every point in space.

We consider oriented lines (1-cells) given parametrically as continuous, one-to-one maps $C:[0,1] \rightarrow \mathcal{M}$. A material line satisfies

$$
(d / d t) C(\sigma, t)=\mathbf{u}(C(\sigma, t), \circ t)
$$

for $\sigma \in[0,1]$ and $t \in \mathbb{R}$. The circle "o" means that we interpret equation (3) in the Stratonovich sense. The (forward) Ito equation $(d / d t) C(\sigma, t)=\mathbf{u}(C(\sigma, t), t)$ equivalent to (3) has the mean changed to $\bar{u}_{i}^{*}(\mathbf{x}, t)=\bar{u}_{i}(\mathbf{x}, t)+\left.(1 / 2)\left(\partial / \partial x^{k}\right) D_{i k}\left(\mathbf{x}, \mathbf{x}^{\prime} ; t\right)\right|_{\mathbf{x}^{\prime}=\mathbf{x}}([31]$, section 3.4. $)$ If $\mathcal{M}=\mathbb{R}^{d}$ or $\mathbb{T}^{d}$ and if $\mathbf{u}(\mathbf{x}, t)$ is a homogeneous random field, then the Ito and Stratonovich interpretations of equation (3) are equivalent. Now let $P_{\mathbf{u}}[C, t]$ denote the conditional probability distribution of lines for a fixed velocity realization $\mathbf{u}$. This distribution satisfies a stochastic Liouville equation:

$$
(d / d t) P_{\mathbf{u}}[C, t]=-\int_{0}^{1} d \sigma \frac{\delta}{\delta C_{i}(\sigma)}\left(u_{i}(C(\sigma), \circ t) P_{\mathbf{u}}[C, t]\right)
$$

Equation (44) is a direct consequence of equation (3) and must also be interpreted in the Stratonovich sense. It is formally equivalent to the Ito equation:

$$
\begin{array}{rl}
(d / d t) P_{\mathbf{u}}[C, t]=-\int_{0}^{1} & d \sigma \frac{\delta}{\delta C_{i}(\sigma)}\left(\left[\bar{u}_{i}^{*}(C(\sigma), t)+\widetilde{u}_{i}(C(\sigma), t)\right] P_{\mathbf{u}}[C, t]\right) \\
& +\frac{1}{2} \int_{0}^{1} d \sigma \int_{0}^{1} d \sigma^{\prime} \frac{\delta^{2}}{\delta C_{i}(\sigma) \delta C_{j}\left(\sigma^{\prime}\right)}\left(D_{i j}\left(C(\sigma), C\left(\sigma^{\prime}\right) ; t\right) P_{\mathbf{u}}[C, t]\right) .
\end{array}
$$


Averaging equation (5) over the Gaussian ensemble of velocities $\widetilde{\mathbf{u}}$ yields a functional FokkerPlanck equation for distributions in the space of free lines $C$ on the manifold $\mathcal{M}$ :

$$
\begin{array}{rl}
(d / d t) P[C, t]=-\int_{0}^{1} & d \sigma \frac{\delta}{\delta C_{i}(\sigma)}\left(\left[\bar{u}_{i}^{*}(C(\sigma), t) P[C, t]\right)\right. \\
& +\frac{1}{2} \int_{0}^{1} d \sigma \int_{0}^{1} d \sigma^{\prime} \frac{\delta^{2}}{\delta C_{i}(\sigma) \delta C_{j}\left(\sigma^{\prime}\right)}\left(D_{i j}\left(C(\sigma), C\left(\sigma^{\prime}\right) ; t\right) P[C, t]\right) .
\end{array}
$$

The first term on the righthand side represents a drift with the mean velocity $\overline{\mathbf{u}}^{*}$ and the second term represents a diffusion arising from the velocity covariance D. Similar diffusions on the path- and loop-spaces of a manifold $\mathcal{M}$ have been much studied, motivated in part by questions from quantum field theory [32, 33].

The above considerations are rigorously justifiable for the case of a Lipschitz velocity with $\alpha=1$ but are only formal when $\alpha<1$. A more careful (and also more physically realistic) approach in the latter case is to replace the advecting velocity u with a "coarse-grained" or smoothed velocity $\mathbf{u}_{\lambda}=\varphi_{\lambda} * \mathbf{u}$, by convolution with a smooth filter kernel $\varphi_{\lambda}(\mathbf{r})=\lambda^{-d} \varphi(\mathbf{r} / \lambda)$. The length-scale $\lambda$ can be interpreted as a mathematical representation of the viscous cutoff in a true turbulent velocity field [25, 26]. The exact solution of the Liouville equation (4) for such a smoothed velocity is

$$
P_{\mathbf{u}_{\lambda}}\left(d C, t \mid C_{0}, t_{0}\right)=\delta\left(C-\boldsymbol{\xi}_{\lambda}^{t, t_{0}}\left(C_{0}\right)\right) d C
$$

with initial condition $P_{\mathbf{u}_{\lambda}}\left(d C, t_{0} \mid C_{0}, t_{0}\right)=\delta\left(C-C_{0}\right) d C$. Here $\boldsymbol{\xi}_{\lambda}^{t, t^{\prime}}: \mathcal{M} \rightarrow \mathcal{M}$ is the stochastic flow of diffeomorphisms generated by the smoothed velocity-field $\mathbf{u}_{\lambda}$ ([31], section 4.6). Despite (17), a nontrivial diffusion process in line-space can be obtained if the limit $\lambda \rightarrow 0$ is taken appropriately. Consider a "nice" distribution $G_{\rho}(d C)$ which is supported on lines entirely contained in the ball $B(\mathbf{0}, \rho)$ of radius $\rho$ at the origin $\mathbf{0}$ and take the weak limit

$$
\lim _{\rho \rightarrow 0} \lim _{\lambda \rightarrow 0} \int G_{\rho}\left(d C_{0}^{\prime}\right) \int P_{\mathbf{u}_{\lambda}}\left(d C, t \mid C_{0}+C_{0}^{\prime}, t_{0}\right) \Psi(C)=\int P_{\mathbf{u}}\left(d C, t \mid C_{0}, t_{0}\right) \Psi(C)
$$

for bounded, continuous functionals $\Psi(C)$ and $t>t_{0}$. In the "weakly compressible regime" [24, 25, 28] - and, in particular, for a divergence-free velocity field satisfying $\boldsymbol{\nabla} \cdot \mathbf{u}=0$ - this 
limit should yield a non-degenerate diffusion. 1 This is a generalization of the phenomenon of spontaneous stochasticity to the turbulent advection of lines, with initial line $C_{0}$ at time $t_{0}$ splitting into a random ensemble of lines $C$ at time $t$.

As for the case of smooth advection, an unconditional diffusion satisfying equation (6) may be obtained by averaging over the velocity $\mathbf{u}$. The instantaneous realizations $C$ of this diffusion process should be fractal objects when the advecting velocity is Hölder continuous with exponent $0<\alpha<1$ and rigorous estimates of their Hausdorff dimensions would be of much interest. These questions may also be addressed numerically using Lagrangian Monte Carlo techniques [34, 35, 36]. In such a study, the material line $C(t)$ would be represented by a discrete approximation $C_{N}(t)$ constructed from $N+1$ Lagrangian particles $\mathbf{x}_{a}(t), a=0, \ldots, N$ :

$$
C_{N}(\sigma, t)=\left(1-\theta_{N}(\sigma)\right) \mathbf{x}_{a_{N}(\sigma)}(t)+\theta_{N}(\sigma) \mathbf{x}_{a_{N}(\sigma)+1}(t)
$$

Here $a_{N}(\sigma)=[N \sigma]$ with $[x]$ the greatest integer less than or equal to $x$ (modulo $N$ for loops) and $\theta_{N}(\sigma)=(N \sigma)$ where $(x)=x-[x]$ is the fractional part of $x$. Thus, (11) corresponds to a piecewise-linear curve with linear segments connecting the successive Lagrangian particles. So long as $\delta_{N}(t)=\max _{a}\left|\mathbf{x}_{a}(t)-\mathbf{x}_{a+1}(t)\right| \lesssim \lambda$, the discrete approximation $C_{N}(t)$ represents well the material line $C(t)$ and the approximation becomes better as $N \rightarrow \infty$ and $\delta_{N}(t) \ll \lambda$. However, the same is not true in the opposite limit, where $\lambda \ll \delta_{N}(t)$. The phenomenon of "spontaneous stochasticity" for a rough velocity field makes it very doubtful that material lines even exist if the limit $\lambda \rightarrow 0$ is taken before evolving in time and an initial line then presumably "explodes"

\footnotetext{
${ }^{1}$ We note that the same diffusion process should also be obtained by using Duhamel's formula to solve equation (5) as the Ito integral

$$
P_{\mathbf{u}}[C, t]=S_{0}^{*}\left(t, t_{0}\right) P\left[C, t_{0}\right]-\int_{t_{0}}^{t} d t^{\prime} S^{*}\left(t, t^{\prime}\right) \int_{0}^{1} d \sigma \frac{\delta}{\delta C_{i}(\sigma)}\left(\left[\widetilde{u}_{i}\left(C(\sigma), t^{\prime}\right)\right] P_{\mathbf{u}}\left[C, t^{\prime}\right]\right),
$$

where $S_{0}^{*}\left(t, t^{\prime}\right)=\operatorname{Texp}\left[\int_{t_{0}}^{t} d t^{\prime} \mathcal{A}^{*}\left(t^{\prime}\right)\right]$ and

$$
\mathcal{A}^{*}(t)=-\int_{0}^{1} d \sigma \frac{\delta}{\delta C_{i}(\sigma)}\left(\left[\bar{u}_{i}^{*}(C(\sigma), t) \cdot\right)+\frac{1}{2} \int_{0}^{1} d \sigma \int_{0}^{1} d \sigma^{\prime} \frac{\delta^{2}}{\delta C_{i}(\sigma) \delta C_{j}\left(\sigma^{\prime}\right)}\left(D_{i j}\left(C(\sigma), C\left(\sigma^{\prime}\right) ; t\right) \cdot\right)\right.
$$

is the Fokker-Planck operator of the (mean) diffusion in line-space. Equation (9) can be solved iteratively to generate a representation $P_{\mathbf{u}}[C, t]=S_{\mathbf{u}}^{*}\left(t, t_{0}\right) P\left[C, t_{0}\right]$ as a Wiener chaos expansion in white-noise $\widetilde{\mathbf{u}} ;$ cf. [28, 29].
} 
into a disconnected cloud of particles at any time $t>0$. Thus, the velocity smoothing in (8) appears to be necessary to define appropriately a line-diffusion for a rough (Hölder) velocity. Alternatively, a stochastic regularization might be employed that adds a white-noise $\kappa d W(t)$ to the evolution equation of Lagrangian particles [30].

We now turn to the dual problem of a passive vector (1-form) A advected by the velocity $\mathbf{u}=\overline{\mathbf{u}}+\widetilde{\mathbf{u}}:$

$$
\partial_{t} \mathbf{A}(\mathbf{x}, t)+(\mathbf{u}(\mathbf{x}, \circ t) \cdot \nabla) \mathbf{A}(\mathbf{x}, t)+(\boldsymbol{\nabla} \mathbf{u}(\mathbf{x}, \circ t)) \mathbf{A}(\mathbf{x}, t)=\mathbf{0}
$$

This stochastic equation is interpreted again in the Stratonovich sense. Equation (12) for $d=3$ is equivalent by vector calculus identities to $\partial_{t} \mathbf{A}+\boldsymbol{\nabla}(\mathbf{u} \cdot \mathbf{A})-\mathbf{u} \times(\boldsymbol{\nabla} \times \mathbf{A})=\mathbf{0}$. The latter has the form of Ohm's law,

$$
\mathbf{E}+\mathbf{u} \times \mathbf{B}=\eta \mathbf{J}
$$

in the ideal limit of zero resistivity $(\eta=0)$ for an electric field $\mathbf{E}=-\partial_{t} \mathbf{A}-\boldsymbol{\nabla}(\mathbf{u} \cdot \mathbf{A})$ and magnetic field $\mathbf{B}=\boldsymbol{\nabla} \times \mathbf{A}$ given by a vector potential $\mathbf{A}$, with the electric current $\mathbf{J}=\boldsymbol{\nabla} \times \mathbf{B}$. Taking the curl of (13) yields an induction equation $\partial_{t} \mathbf{B}=\nabla \times(\mathbf{u} \times \mathbf{B})+\eta \triangle \mathbf{B}$ for the magnetic field. With this interpretation, the passive vector equation was introduced by Kazantsev [37] as a soluble model of the kinematic dynamo. (See also [38, 39, 40].) The "circulation" (or "holonomy") of $\mathbf{A}$ along $C$ is defined in Lagrangian form as

$$
\Phi_{L}(C, t)=\int_{C(t)} \mathbf{A}(t) \cdot d \mathbf{x}
$$

where $C(t)$ is the material line advected by $\mathbf{u}(t)$ which started as line $C$ at the initial time $t=t_{0}$. Conservation of "circulation", $(d / d t) \Phi_{L}(C, t)=0$, follows formally from (12) for any space dimension $d \geq 1$. It is rigorously true for the case of a smooth advecting velocity with $\alpha=1$ ([31], section 4.9). If $C$ is a closed loop (1-cycle), then the line-integral (14) represents gauge-invariant magnetic flux and the conservation law corresponds to Alfvén's theorem [20].

We now consider the generalization of this result for $\alpha<1$. For this purpose, it is useful to 
reformulate the passive vector equation (12) as a passive scalar in line-space:

$$
\partial_{t} \Phi_{E}(C, t)+\int_{0}^{1} d \sigma\left[\bar{u}_{i}(C(\sigma), t)+\widetilde{u}_{i}(C(\sigma), \circ t)\right] \frac{\delta}{\delta C^{i}(\sigma)} \Phi_{E}(C, t)=0 .
$$

In this equation, $\Phi_{E}(C, t)=\int_{C} \mathbf{A}(t) \cdot d \mathbf{x}$ is the Eulerian circulation of $\mathbf{A}$ along a fixed (nonadvected) line $C$. An exactly analogous reformulation of the incompressible Euler equation (as an active scalar in loop-space) was advanced some time ago by Migdal [41]. Note that conservation of circulations is just the formal solution of (15) by the method of characteristics. We shall take the equation (15) as our primitive formulation of the passive vector; one of the immediate advantages is that we can avoid (for the moment) the question how to define line-integrals over fractal lines. We then convert (15) to Ito formulation:

$$
\begin{aligned}
\partial_{t} \Phi_{E}(C, t)=-\int_{0}^{1} d \sigma\left[\bar{u}_{i}^{*}(C(\sigma), t)+\widetilde{u}_{i}(C(\sigma), t)\right] \frac{\delta}{\delta C^{i}(\sigma)} \Phi_{E}(C, t) \\
+\frac{1}{2} \int_{0}^{1} d \sigma \int_{0}^{1} d \sigma^{\prime} D_{i j}\left(C(\sigma), C\left(\sigma^{\prime}\right), t\right) \frac{\delta^{2}}{\delta C^{i}(\sigma) \delta C^{j}\left(\sigma^{\prime}\right)} \Phi_{E}(C, t)
\end{aligned}
$$

This stochastic equation is solved by the method of LeJan and Raimond [28, 29] (cf. footnote $\# 1$, writing it as a (backward) Ito integral and iterating to obtain $\Phi_{E}(C, t)=S_{\mathbf{u}}\left(t, t^{\prime}\right) \Phi_{E}\left(C, t^{\prime}\right)$, where the Markov operator semi-group $S_{\mathbf{u}}\left(t, t^{\prime}\right)$ is defined by a Wiener chaos expansion. More intuitively, this solution is expressed as

$$
\Phi_{E}(C, t)=\int P_{\mathbf{u}}\left(d C^{\prime}, t^{\prime} \mid C, t\right) \Phi_{E}\left(C^{\prime}, t^{\prime}\right), \quad t^{\prime}<t
$$

in terms of the turbulent diffusion of lines (backward in time). We see that the circulations are not conserved, except on average. This is precisely the "martingale property" that was conjectured (for solutions of incompressible Euler equations) in [18]. Note that this property imposes an irreversible arrow of time, since Eulerian circulations are given as averages over their past values, not future ones. This "generalized Alfvén theorem" should be related to dynamo action in the Kazantsev model [37, 38, 39, 40]. In the physical context of the dynamo, there is an additional resistive term $\eta \oint_{C} d x_{j} \partial_{i} \frac{\delta}{\delta \sigma_{i j}(\mathbf{x})} \Phi_{E}(C, t)$ on the righthand side of (15), where $\delta / \delta \sigma_{i j}(\mathbf{x})$ is the "area derivative" in the loop calculus of Migdal [41]. This term breaks 
time-reversal symmetry and should select the backward-martingale solution (17) in the ideal limit $\eta \rightarrow 0$. Of course, it cannot be ruled out a priori that the $\eta \rightarrow 0$ limit of the resistive regularization and the $\lambda \rightarrow 0$ limit for regularized velocity, as in (8), shall yield distinct weak solutions of the loop-equation (15), as occurs in the intermediate compressibility regime of the passive scalar problem [24, 25, 26, 28].

These results can be generalized to turbulent diffusion processes of higher-dimensional material objects, $k$-dimensional oriented submanifolds of $\mathcal{M}$ or $k$-cells $C^{k}(t)$. The dual object is the passive $k$-form $\omega^{k}$, which satisfies (in Stratonovich sense)

$$
\partial_{t} \omega^{k}+L_{\mathbf{u}} \omega^{k}=0
$$

with $L_{\mathbf{u}}$ the Lie-derivative along the vector field $\mathbf{u}$ ([31], section 4.9). This equation is formally equivalent to conservation of the integral invariants

$$
I\left(C^{k}, t\right)=\int_{C^{k}(t)} \omega^{k}(t)
$$

for any $k$-cell $C^{k}(t)$ comoving with $\mathbf{u}[42$. Then $k=0$ is the passive scalar, $k=1$ the passive vector and $k=d$ the passive density [24]. A theory similar to that developed here for $k=1$ applies for any integer $k$. A unified approach to all these results is to consider directly the turbulent diffusion of the Lagrangian flow maps $\boldsymbol{\xi}^{t, t^{\prime}}$, which satisfy the stochastic equation

$$
(d / d t) \boldsymbol{\xi}^{t, t^{\prime}}(\mathbf{a})=\mathbf{u}\left(\boldsymbol{\xi}^{t, t^{\prime}}(\mathbf{a}), \circ t\right), \quad \boldsymbol{\xi}^{t^{\prime}, t^{\prime}}(\mathbf{a})=\mathbf{a}
$$

In this framework one can derive for the distribution $P_{\mathbf{u}}[\boldsymbol{\xi}, t]$ on maps exact analogues of the Liouville equation, in Stratonovich form (44) or Ito form (5). It is natural to formulate the problem as an infinite-dimensional diffusion in the Hilbert space $\mathcal{H}=L^{2}\left(\mathcal{M}, \mathbb{R}^{d}\right)$. It is known for the cases $\mathcal{M}=\mathbb{R}^{d}$ or $\mathbb{T}^{d}$ that the semigroup $S(\mathcal{M})$ of Borel volume-preserving maps is a closed subset of this Hilbert space, and that the group $G(\mathcal{M})$ of volume-preserving diffeomorphisms is dense in $S(\mathcal{M})$ for the $L^{2}$-topology [43]. This construction is a close analogue of the "generalized Euler flows" of Brenier, but for the Cauchy initial-value problem. 
To summarize: We have outlined an approach to the study of material lines in a model of turbulent flow at infinite Reynolds number and to the dual problem of a passive vector in the same flow. The main conclusions are (1) that a non-degenerate diffusion should exist for material lines, generalizing the phenomenon of "spontaneous stochasticity" of material points, and (2) that the Kelvin/Alfvén theorem on conservation of circulations should generalize to a "martingale property". Although the approach sketched here depends heavily on the whitenoise character of the velocity field in time, we expect that similar results hold for more realistic velocity ensembles with the crucial property that realizations are rough (Hölder continuous) in space. See [18, 21] for related rigorous results on the solutions of incompressible fluid equations. The two properties discussed in the context of this model problem should be an essential feature of real fluid turbulence in the high Reynolds number limit.

Acknowledgements. We thank S. Chen, M. Chertkov, L. Chevillard, R. Ecke, C. Meneveau, K. R. Sreenivasan and E. T. Vishniac for useful conversations. This work was supported by the NSF grant \# ASE-0428325 at the Johns Hopkins University and by the Center for Nonlinear Studies at Los Alamos National Laboratory, where the research was begun. 


\section{References}

[1] G. K. Batchelor, "The effect of homogeneous turbulence on material lines and surfaces," Proc. Roy. Soc. Lond. A 213 349-366 (1952).

[2] G. I. Taylor. "Observations and speculations on the nature of turbulence motion (1917)", in: Scientific Papers of Sir Geoffrey Ingram Taylor, vol.2, ed. G.K. Batchelor (Cambridge Univ. Press, 1971), p.69.

[3] G. I. Taylor and A. E. Green, "Mechanism of the production of small eddies from large ones", Proc. R. Soc. Lond. A, 158, 499-521 (1937).

[4] G. I. Taylor, "Production and dissipation of vorticity in a turbulent fluid," Proc. R. Soc. Lond. A 164 15-23 (1938).

[5] G. K. Batchelor, "On the spontaneous magnetic field in a conducting liquid in turbulent motion," Proc. Roy. Soc. Lond. A 201 405-416 (1950).

[6] P. G. Saffman, "On the fine-scale structure of vector fields advected by a turbulent fluid," J. Fluid Mech. 16545 (1963).

[7] L. Onsager, "Statistical hydrodynamics," Nuovo Cimento, 6 279-287 (1949).

[8] G. Parisi and U. Frisch, "On the singularity structure of fully developed turbulence," Turbulence and Predictability in Geophysical Fluid Dynamics, eds. M. Gil, R. Benzi and G. Parisi (North-Holland, 1985), pp.84-88.

[9] G. L. Eyink, "Besov spaces and the multifractal hypothesis," J. Stat. Phys. 78 353-375 (1995).

[10] B. B. Mandelbrot, "Géométrie fractale de la turbulence. Dimension de Hausdorff, dispersion et nature des singularités du mouvement des fluides," Comptes Rendus (Paris) $\mathbf{2 8 2} \mathbf{A}$ 119-120 (1976). 
[11] K. R. Sreenivasan and C. Meneveau, "The fractal facets of turbulence," J. Fluid Mech. $173357-386$ (1986).

[12] J. C. H. Fung and J. C. Vassilicos, "Fractal dimensions of lines in chaotic advection," Phys. Fluids A 31433 (1991).

[13] E. Villermaux and Y. Gagne, "Line dispersion in homogeneous turbulence: stretching, fractal dimensions, and micromixing," Phys. Rev. Lett. 73 252-255 (1994).

[14] D. Kivotides, "Geometry of turbulent tangles of material lines," Phys. Lett. A 318 574-578 (2003).

[15] F. C. G. A. Nicolleau and A. Elmaihy, "Study of the development of three-dimensional sets of fluid particles and iso-concentration fields using kinematic simulation," J. Fluid Mech. $517229-249$ (2004).

[16] H. Helmholtz, "Über Integrale der hydrodynamischen Gleichungen welche den Wirbelbewegungen entsprechen", Crelles Journal, 55 25-55 (1858).

[17] W. Thomson (Lord Kelvin), "On vortex motion", Trans. Roy. Soc. Edin., 25, 217-260 (1869).

[18] G. L. Eyink, "Turbulent cascade of circulations," Comptes Rendus Physique, 7 449-455 (2006).

[19] S. Chen, G. L. Eyink, Z. Xiao, and M. Wan, "Is the Kelvin theorem valid for high-Reynoldsnumber turbulence?" Phys. Rev. Lett. 97144505 (2006).

[20] H. Alfvén, "On the existence of electromagnetic-hydrodynamic waves," Arkiv f. Mat., Astron. o. Fys. 29B 1-7 (1942)

[21] G. L. Eyink and H. Aluie, "The breakdown of Alfvén's theorem in ideal plasma flows: necessary conditions and physical conjectures," Physica D 223 82-92 (2006). 
[22] R. H. Kraichnan, "Small-scale structure of a scalar field convected by turbulence," Phys. Fluids 11 945-953 (1968).

[23] D. Bernard, K. Gawȩdzki, and A. Kupiainen, "Slow modes in passive advection," J. Stat. Phys. 90 519-569 (1998).

[24] K. Gawędzki and M. Vergassola, "Phase transition in the passive scalar advection," Physica D 138 63-90 (2000).

[25] W. E and E. Vanden-Eijnden, "Generalized flows, intrinsic stochasticity, and turbulent transport," Proc. Nat. Acad. Sci. (USA) 97 8200-8205 (2000).

[26] W. E and E. Vanden-Eijnden, "Turbulent Prandtl number effect on passive scalar advection," Physica D 152 636-645 (2001).

[27] Y. Le Jan and O. Raimond, "Solutions statistiques fortes des équations différentielles stochastiques," C. R. Acad. Sci. Paris 327, Série I, 893-896 (1998)

[28] Y. Le Jan and O. Raimond, "Integration of Brownian vector fields," Ann. Prob. 30 826-873 (2002).

[29] Y. Le Jan and O. Raimond, "Flows, coalescence and noise," Ann. Prob. 32 1247-1315 (2004).

[30] G. Falkovich, K. Gawędzki \& M. Vergassola, "Particles and fields in fluid turbulence," Rev. Mod. Phys. 73 913-975 (2001).

[31] H. Kunita, Stochastic Flows and Stochastic Differential Equations. (Cambridge University Press, Cambridge, 1990).

[32] B. Driver and M. Röckner, "Construction of diffusions on path and loop spaces of compact Riemannian manifolds," Compt. Rend. Acad.Sci., Serie I 316 603-608 (1992). 
[33] R. Léandre, "Analysis on loop spaces and topology," Mathematical Notes 72 212-229 (2002).

[34] U. Frisch, A. Mazzino, and M. Vergassola, "Intermittency in passive scalar advection," Phys. Rev. Lett. 80 5532-5534 (1998).

[35] U. Frisch, A. Mazzino, A. Noullez and M. Vergassola, "Lagrangian method for multiple correlations in passive scalar advection," Phys. Fluids 11 2178-2186 (1999).

[36] O. Gat, I. Procaccia, and R. Zeitak, "Anomalous scaling in passive scalar advection: Monte Carlo Lagrangian trajectories," Phys. Rev. Lett. 80 5536-5539 (1998).

[37] A. P. Kazantsev, "Enhancement of a magnetic field by a conducting fluid," Sov. Phys. JETP 26 1031-1034 (1968).

[38] M. Vergassola, "Anomalous scaling for passively advected magnetic fields," Phys. Rev. E 53 R3021-R3024 (1996).

[39] D. Vincenzi, "The Kraichnan-Kazantsev dynamo," J. Stat. Phys. 106 1073-1091 (2002)

[40] A. Celani, A. Mazzino, and D. Vincenzi, "Magnetic field transport and kinematic dynamo effect: a Lagrangian interpretation,” Proc. R. Soc. A 462 137-147 (2006).

[41] A.A. Migdal, "Loop equation in turbulence," hep-th/9303130; "Turbulence as statistics of vortex cells," hep-th/9306152; "Loop equation and area law in turbulence," hep-th/9310088

[42] R. Abraham, J. E. Marsden, and T. Ratiu, Manifolds, Tensor Analysis, and Applications. Applied Mathematical Sciences, vol. 75. (Springer-Verlag, Berlin, 1983).

[43] Y. Brenier, "Topics on hydrodynamics and volume preserving maps," in: Handbook of Mathematical Fluid Dynamics II. (North-Holland, Amsterdam, 2003), pp.55-86. 